\title{
INCORPORATING RELIABILITY CALCULATIONS IN ROUTINE NETWORK PLANNING: THEORY AND PRACTICE
}

\author{
J.G. SLOOTWEG*, P.M. VAN OIRSOUW** \\ *Essent Netwerk B.V., P.O. Box 856, 5201 AW, 's-Hertogenbosch \\ **Phase to Phase B.V., P.O. Box 100, 6800 AC, Arnhem The \\ Netherlands
}

\begin{abstract}
SUMMARY
This paper describes the implementation of reliability calculations into the routine network planning of a distribution network operator. Firstly, actual developments putting this issue on the agenda are described. Then, it is argued why medium voltage (MV) networks form the logical starting point. Hereafter, the basics of reliability calculations and the approach towards reliability calculations, as it is implemented in the MV network analysis program Vision, are elaborated upon. Finally, the practical implementation and applicability of reliability calculations is discussed. It is argued that the results of reliability calculations must be interpreted carefully, but that they can nevertheless be of good use in specific situations.
\end{abstract}

\section{INTRODUCTION}

The availability of electric power is a prerequisite for the functioning of modern societies. These are heavily dependent on mass transportation, information and communication technologies, etc. which nearly always use electricity as their source of energy. The public and political awareness of the importance of a reliable electricity supply is increasing. Explanations for this are, amongst others, the large blackouts that have recently occurred in several countries, as well as the intense attention drawn by the electricity sector due to the restructuring that is currently taking place throughout the world.

With the restructuring of the electricity sector, the transmission and distribution networks tend to be considered a natural monopoly. Therefore, regulating bodies are created in order to prevent network operators from abusing their monopoly power. These protect customers that depend on the networks for the supply of a vital resource. Although the regulatory approaches adopted by these bodies vary, their aim is to increase in some way or the other the effectiveness and efficiency of the network operators. The goal is to achieve both lower tariffs as well as an increase in supply reliability for customers connected to the network.

It can be easily imagined that the increasing political and public expectations, together with the income decreases being faced by network operators, lead to a pressure on cost. Consequently, it becomes evermore important to justify any expenditure by showing that it either prevents a larger expenditure in the future, or that it is an efficient way of serving the business values of the network owner. An example of the first type of expenditure is the painting of high voltage towers in order to prevent corrosion of the tower steel leading to more expensive maintenance in the future (such as sand- blasting and steel bar replacements). An example of the second kind of expenditure could be the use of short circuit detectors in order to accelerate the restoration process after a disconnection due to a fault, thus increasing reliability of supply.

In most cases, reliability of supply will be amongst the business values of a network owner. Thus, in order to investigate whether proposed expenditures for increasing the reliability of supply are indeed effective and efficient, it should be possible to assess their impact on the reliability of supply. The quality regulation approaches presently being adopted by many regulators reinforce this necessity, because they establish a relation between reliability of supply on the one hand and the tariff that a network operator is allowed to charge to its customers on the other. Thus, in order to evaluate possible measures for increasing supply reliability under a quality regulation regime, both the cost and the benefits must be known. For investigating the latter, reliability calculations can be used.

The topic of this paper is the incorporation of reliability calculations in routine network planning, a goal that is motivated by the aforementioned developments. First, a general overview over the reliability of supply in The Netherlands will be given and it will be shown that based on this, the medium voltage (MV) networks form the natural starting point for the incorporation of reliability calculations in network planning. Then, a brief introduction towards the area of reliability calculations will be given and the basics of Vision, a network analysis program used widely in The Netherlands, will be described. Finally, some practical aspects of the incorporation of reliability calculations in routine network planning will be treated.

\section{RELIABILITY OF SUPPLY IN THE NETHERLANDS}

Several indicators for measuring reliability of supply exist. The ones most frequently used are [1]:

- System Average Interruption Frequency Index (SAIFI)

$S A I F I=\frac{\text { Total number of customer interruptions }}{\text { Total number of customers served }}$

- System Average Interruption Duration Index (SAIDI)

$S A I D I=\frac{\sum \text { Customer interruption durations }}{\text { Total number of customers served }}$

- Customer Average Interruption Duration Index (CAIDI)

$C A I D I=\frac{\sum \text { Customer interruption durations }}{\text { Total number of customer interruptions }}$ 
These indicators are widely used for comparing network operators and may also be incorporated in quality regulation approaches at present being adopted by some regulators [2-3]. In figure 1 , the development of the SAIDI over the last 30 years is depicted for The Netherlands [4]. In this figure, the total SAIDI is divided into separate contributions from high voltage $(\mathrm{HV}, \geq 50 \mathrm{kV})$, medium voltage (MV, $<50 \mathrm{kV}-1 \mathrm{kV})$ and low voltage $(\mathrm{LV},<1 \mathrm{kV})$. From the figure, a number of conclusions can be drawn:

- The contribution of LV outages to the total SAIDI is relatively low and steady.

- MV outages have the largest contribution to the total SAIDI, and their contribution fluctuates averagely.

- The contribution of HV outages varies between very small (e.g. 1986 and 1992) to very large (e.g. 1990 and 1997).

These observations can be explained as follows. In the SAIDI score, both the number of outages as well as the number of affected customers play a role. The higher the number of outages incorporated in a (part of a total) SAIDI score, the steadier it will be over time.

The contribution of LV is built by very many relatively small outages and is hence quite steady. The contribution of MV outages is built by less outages each affecting more customers, and is therefore less steady. Finally, the contribution of $\mathrm{HV}$ outages (that occur rather seldom due to the high degree of redundancy in HV networks) is built by a small number of large outages, explaining its high variability over the years.

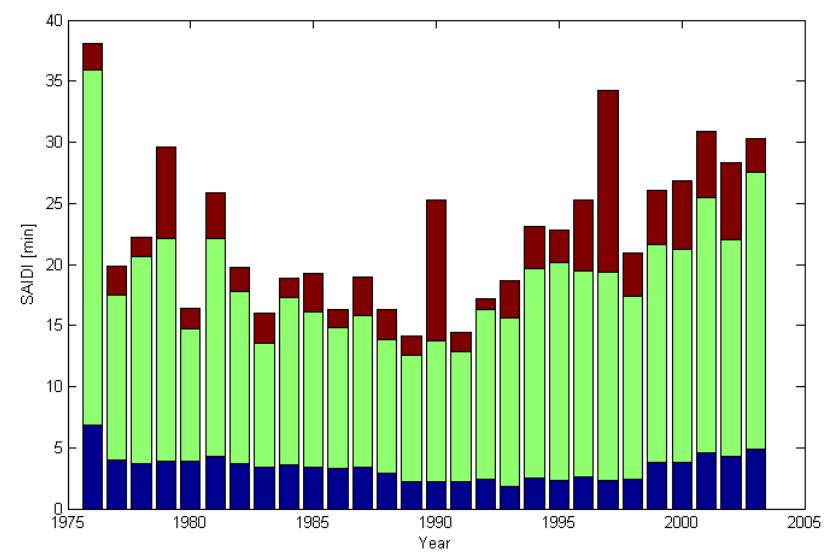

Figure 1. Development of SAIDI in The Netherlands; total SAIDI and contribution of HV, MV and LV outages (from above)

\section{RELIABILITY CALCULATIONS}

\section{Basics of reliability calculations}

The area of reliability calculations has been intensively studied for several decades. Many books, theses and papers on the topic have appeared and it is not assumed that this flow will dry up in the near future. Subjects being discussed are for instance various modelling and calculation approaches, the impact of input parameter variations on the calculation results (i.e. sensitivity analysis), etc. [5-8]

Nevertheless, the basics of reliability calculations are quite easy to understand intuitively. The required inputs are:

- the topology of the network to be studied

- the network component characteristics ( "name plate" data such as line and cable lengths and component ratings, electrical characteristics, as well as failure rates, maintenance intervals and the duration of repairs)

- the parameters characterising the restoration process Using these inputs, an algorithm calculates for each component outage whether any loads are no longer served and if so, how often and how long this will be the case. By appropriately summing the outage frequencies and durations for all loads, reliability indicators for the individual loads as well as for the network can be computed.

However, although principally straightforward, practical implementation of reliability calculations requires advanced mathematical approaches. Tasks such as finding the optimal way of supply restoration by reconfiguration of the network without overloading any component and while taking into account the possibility of using of emergency units require sophisticated algorithms. Sometimes, even uncertainties in the input data, particularly in failure statistics, progress of the restoration process and network loading can be allowed for, for instance by using Monte Carlo simulations. In the next section, as an example the approaches implemented in one software package will be explained in more detail.

By translating the assumed effect of some measure into changes in the input parameters for the calculations, reliability calculations can be used to study the impact of possible measures on supply reliability. A change in network topology (e.g. adding extra connections in order to increase the level of redundancy) directly translates into a corresponding change in the network model's topology. A change in maintenance or replacement philosophy of a certain component is reflected in the failure rate of that component. Finally, measures affecting the restoration process (such as the commitment of extra personnel or additional training) is reflected in the parameters characterising the restoration process.

\section{Reliability calculations using Vision}

As can be seen in figure $1, \mathrm{MV}$ outages contribute the largest part to the SAIDI score in The Netherlands. Hence, these networks form the logical starting point for the incorporation of reliability calculations in routine network planning. For the analysis of MV networks, the program Vision is widely used in The Netherlands. The capabilities of this program and its approach towards reliability calculations will now be described.

Program description. Vision is a high-grade tool for $\mathrm{HV}$ and MV network analysis. Its calculation modules comprise load flow, short circuit, fault analysis, protection analysis, reliability calculation and cost analysis. Special applications are transmission analysis, fault location and pseudo state estimation. In many cases the Vision software is integrated in a Geographical Information System, enabling a two-way information exchange. 
User convenience and practical applicability of results have always been central to the development of Vision. As a result, the software complies to the Windows standard, concerning both look and feel and communication with Microsoft Office products, keeping the object information intact.

Reliability calculation approach. The Vision Reliability analysis module follows an analytical approach. All possible failures and their remedial and restoration actions are evaluated. All components fail according to their specified failure rates. Also common cause failures, failures during maintenance and switches failing to operate are modelled. The Vision reliability module is modelled as follows:

- a component (source, generator, load, node, branch) fails

- the commonly protected group of components is switched off; also other groups of components may be switched off consequently

- the failure frequency is added to all of the components that are switched off

- for all components and loads the interruption durations are determined by evaluating the restoration process

The interruption duration is calculated by analysing the possible remedial actions until the faulted component is isolated and all other components are back in service again. The distribution network is supposed to be a radially operated meshed network, but also meshed operation as well as nonmeshed layouts are allowed.

The restoration process is modelled as follows:

- a fault is signalled

- the trouble-shooting crew is activated and travels to the defected feeder

- the faulted section or component is located

- the faulted section is isolated

- the feeder circuit breaker is closed to power up the first part of the feeder

- a normally opened switch is closed to power up the last part of the feeder

- if necessary, an emergency generator is used or the component will be repaired

The restoration process flow diagram is illustrated in figure 2 . All above mentioned actions have user defined durations. These durations are first of all company specific, but they also depend on the level of remote sensing and controlling in the network. In the restoration process all defined times are summed and allocated to the components to be restored. Depending on their location in the network, the restoration times may vary in the whole interrupted network section. Mostly, the components in the first part of the feeder will have the shortest restoration time.

The time to locate the faulted section can be a fixed or a variable time, the latter depending on the search method (sequential or binary search) and the number of $\mathrm{MV} / \mathrm{LV}$ transformer stations.

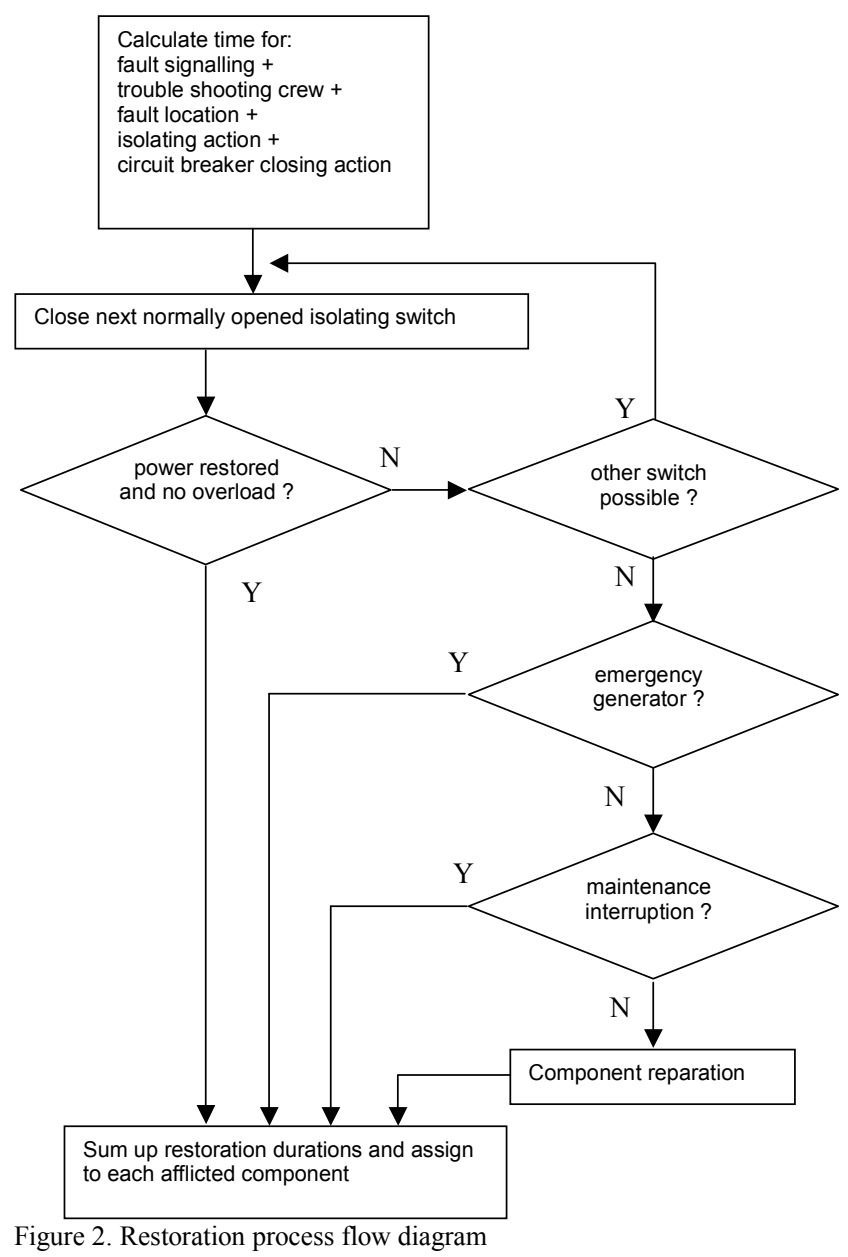

Closing a normally opened switch to restore the last part of the feeder may cause an overload in another part of the network. In those cases, all other possibilities of closing normally opened switches are tried until a satisfactory solution is found.

In most cases the switches closing actions are sufficient to restore all interrupted loads. But in case of a dead end feeder or a faulted node, an emergency generator or a repair action may be needed. The emergency generator operation is limited to the maximum power and operating voltage. Also the possibility to restore power by interrupting a coinciding maintenance operation is evaluated. In all other cases reparation or replacement is necessary.

The interruption duration strongly depends on the company strategy for remedial actions. The repair rate has a relatively small influence. Only in the cases that no remedial action for restoration of delivery is possible, the faulted component has to be replaced or repaired.

After a calculation the following results are available for all components:

- the mean number of component outages per year

- the mean outage duration of the component per outage

- the mean outage duration of the component per year

- the outage probability of the component per year

- the mean amount of energy per year that the load is not served 
Figure 3 illustrates the relationship between the interruption frequency per year and the average interruption duration in minutes per interruption for all failures in a fictitious network. As can be seen in the diagram, a considerable number of components have a relative high interruption frequency. This may be caused by the sum of all failures in a large feeder. This may be considered as a weak spot. One possible improvement could be the addition of an extra protective element such as a circuit breaker, halfway the feeder length. As a result, the number of components with a high interruption frequency reduces.

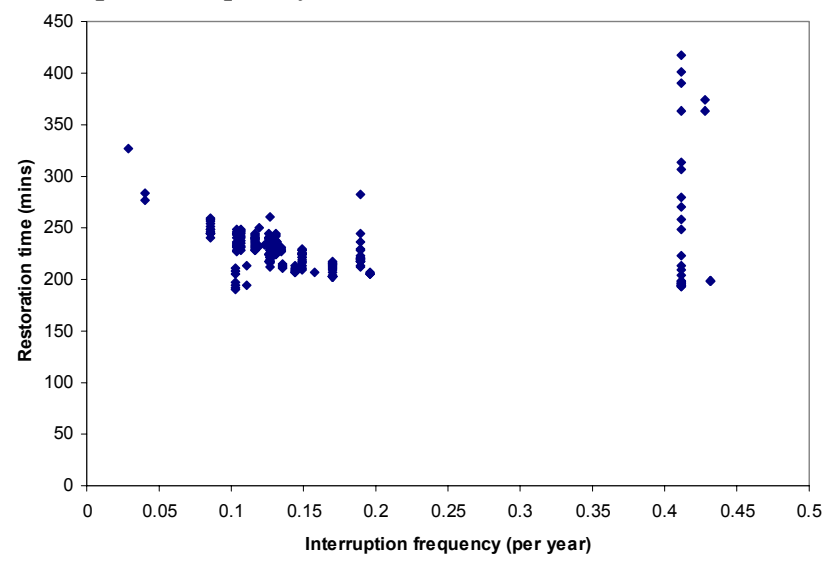

Figure 3. Relation between interruption frequency and restoration time

\section{IMPLEMENTATION OF RELIABILITY CALCULATIONS}

\section{Preconditions}

In order to implement reliability calculations in routine network planning, a number of preconditions must be fulfilled. Firstly, the network planners must be educated in order to give them a general background in reliability calculations, to enable them to fully use the capabilities of their network analysis software and to help them interpreting the obtained results. Due to the number of employees involved, an in-company training in most cases seems advisable.

Secondly, a table of standardized reliability figures must be established, communicated and maintained. The available historical data is often not sufficiently detailed and/or extensive to allow failure rates and repair durations to be directly based on recorded failure statistics. Therefore, the approach here is to use a combination of recorded data, data from other sources (e.g. from scientific literature) and expert judgment. The latter increases the support for the numbers and hence for the calculation results throughout the organization. Parallel to establishing the initial table with standardized figures, registration and reporting of component failures and the restoration process may be adapted in order to improve the accuracy of the figures as well as to notice trends (for instance due to aging of the network or due to the effect of measures that have been undertaken).

\section{Accuracy of results}

As a matter of fact, it has been shown that the results of reliability calculations do not always match the reliability figures observed in reality [5]. There are several reasons for this:

- As mentioned already, it is often difficult to base failure rates on sufficient real data, which may lead to errors in the estimated failure rates and hence in the calculation results.

- Real networks can in some cases be relatively small, so that the reliability observed from year to year can vary quite much and is difficult to compare with the outcome of a reliability calculation, which principally depicts the long year average. The larger the investigated network, the less this effect will occur.

- It is difficult to take differences in reliability between components of the same category (caused by for example age, loading, brand, etc.) into account, although these may heavily affect the outcomes, particularly when studying smaller parts of the system [9].

- The quality of the settings of protection devices has an influence on the number of substations that is effected by an outage. Incorporation of this effect requires a different approach of reliability calculation.

- The restoration process is quite complicated and many factors are involved, which makes it difficult to model.

The last point is illustrated in figures 4 and 5 . In figure 4 , a histogram of the time that passes between the detection of an outage (which can e.g. happen through calls of customers or through remote sensing) and the arrival of the crew in the affected region for a hundred typical MV outages that have occurred at Essent Netwerk B.V. is depicted. In figure 5 for the same outages a scatter plot of the number of affected MV nodes versus the time needed to restore the supply for all customers is depicted. From the figures, it can be concluded that the time between outage detection and crew arrival varies widely and that there is only a weak correlation between number of stations affected and restoration time, although this correlation is often assumed in reliability calculations.

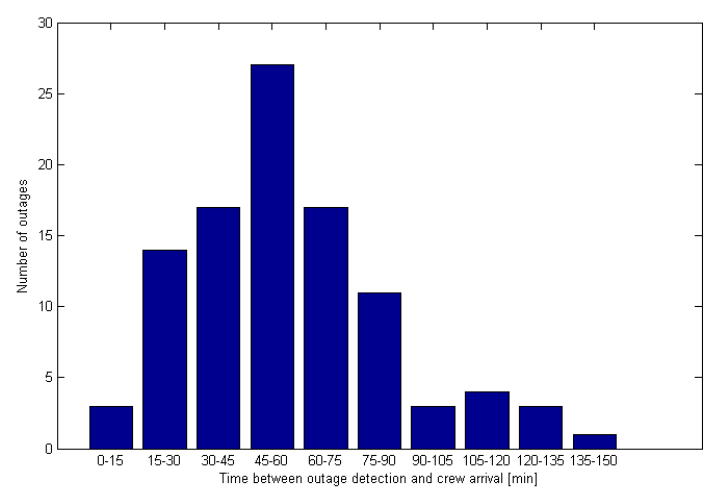

Figure 4. Time passing between outage detection and arrival of crew in affected region for 100 typical MV outages 


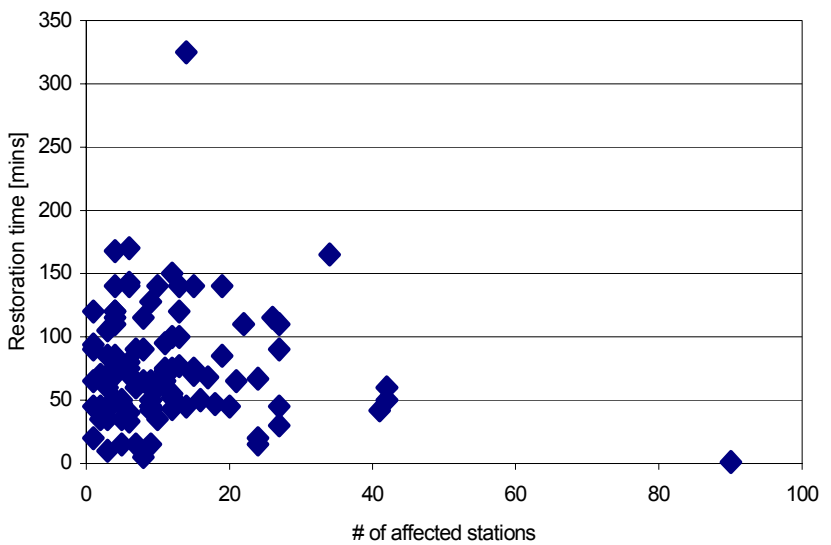

Figure 5. Correlation between number of stations affected and restoration time for 100 typical MV outages

\section{Practical use}

Given the limitations of reliability calculations, the outcomes should be used sensibly. For reasons mentioned in the last section, it is not advisable to consider the calculation results as being an accurate prediction of the expected reliability, particularly not for smaller networks or parts of a network. Nevertheless, if interpreted carefully, the results of reliability calculations can be used fruitfully for several aims, such as:

- Comparison of different configurations of a network

- Comparison of different topologies for network extension and the connection of large customers

- Comparison of the reliability of supply throughout a network in order to find "weak spots"

- The investigation of the impact of (organizational) changes affecting the restoration process

- The investigation of changes in maintenance or replacement policies

Generally spoken, the results of reliability calculations should not be used in an absolute way, but in a relative way, i.e. for comparisons. When one solution or approach turns out to be several times more reliable than another according to the calculation results, there will definitely also be a difference in reality. On the other hand, if the outcome of the reliability calculations only shows a small difference, in reality the difference will be small as well and the option most reliable according to the calculations might even be worse in reality. In this case, reliability aspects should not be used for making a choice and other factors, such as cost, should decide.

\section{CONCLUSIONS AND FURTHER WORK}

It can be concluded that for various reasons, reliability of supply becomes increasingly important. It should therefore be continuously investigated how reliability aspects can be incorporated in routine network planning. It is shown in this paper that this is not at all straightforward, as the reliability observed in reality is influenced by many different factors which are often difficult to model.
It was therefore concluded that the outcomes of reliability calculations should not be used in an absolute sense, i.e. not to predict future reliability figures, but rather for comparisons of various topologies and solutions. Reliability aspects should only be incorporated in the final decision if the calculation results for the alternatives show a significant difference. Otherwise, (only) other factors should decide.

The next step that will be taken is acquiring more insight in the value of reliability calculations in practice. To this end, reliability will be incorporated in a number of network planning decisions, taking into account the various preconditions for a practical implementation as discussed above, in order to see whether the calculation results indeed affect decisions or not. Based on the results of the evaluation a final decision whether to institutionalise reliability calculations or not will be taken.

\section{ACKNOWLEDGEMENT}

Our colleagues Jürgen Mutsaers and Bas Rombouts at Essent Netwerk B.V. are acknowledged for the background information they provided, which proved to be very useful in writing this paper.

\section{REFERENCES}

[1] Transmission and Distribution Subcommittee of the IEEE Power Engineering Society, 2001, IEEE Guide for Electric Power Distribution Reliability Indices, IEEE-SA Standards Board, Piscataway.

[2] www.dte.nl

[3] www.ofgem.gov.uk

[4] KEMA/EnergieNed, 2003, Betrouwbaarheid van elektriciteitsnetten in Nederland in 2003, Arnhem (in Dutch). [5] J.J. Meeuwsen, 1998, Reliability evaluation of electric transmission and distribution systems, Ph.D. thesis TU Delft, Delft.

[6] J. van Casteren, 2003, Assessment of Interruption Costs in Electric Power Systems using the Weibull-Markov Model, Ph.D. thesis Chalmers University of Technology, Göteborg. [7] G.J. Anders, 1990, Probability concepts in electric power systems, John Wiley\&Sons Inc.

[8] J.J. Meeuwsen, W.L. Kling, S.P.J. Rombouts, 1997, “The influence of protective relay schemes on the reliability indices of load points in meshed operated MV networks", CIRED, Birmingham.

[9] R.E. Brown, 2004, "Failure Rate Modeling Using Equipment Inspection Data", IEEE PES General Meeting, Denver. 